\title{
Association between low vitamin D and COVID-19: don't forget the vitamin $D$ binding protein
}

\author{
Marijn M. Speeckaert ${ }^{1,2}$ - Joris R. Delanghe ${ }^{3}$
}

Received: 12 May 2020 / Accepted: 20 May 2020 / Published online: 28 May 2020

C) Springer Nature Switzerland AG 2020

With interest, we read the paper of Ilie et al. about the role of vitamin $\mathrm{D}$ in the prevention and mortality of coronavirus disease-2019 (COVID-19) [1]. A potential association between low mean vitamin $\mathrm{D}$ concentrations in various European countries, and the prevalence and mortality of COVID-19 was observed. The authors focussed mainly on the effect of vitamin D on ACE2 expression in the lungs to explain their findings in patients with severe acute respiratory syndrome coronavirus-2 (SARS-CoV-2). However, we think that also the vitamin D binding protein (DBP), the major transport protein of vitamin $\mathrm{D}$ (at a single binding cleft of the A domain of the protein), may play an underestimated role in the development of acute respiratory syndrome (ARDS) due to SARS-CoV-2 infection.

DBP, formerly known as group-specific component (GCglobulin), is a multifunctional plasma protein with a molecular weight of 52-59 kDa. Besides high-affinity vitamin D binding (resulting in extremely low free concentrations of all vitamin D metabolites), DBP can bind actin, CD44, and annexin A2. This $\alpha_{2}$-globulin plays probably a more crucial role during inflammation than initially thought, as it can significantly enhance the chemotactic activity of neutrophil chemoattractants [2].

During ARDS, a condition associated with extensive tissue damage and cell death, actin (the major cytoskeletal protein) is released into extracellular fluids, leading to lung inflammation. An increased blood neutrophil adhesion and migration, and a stimulated production of inflammatory factors by pulmonary monocytes are involved in this process [3]. SARS-CoV-2 infection has also been associated with

Marijn M. Speeckaert

Marijn.Speeckaert@ugent.be

1 Department of Nephrology, Ghent University Hospital, Corneel Heymanslaan 10, 9000 Ghent, Belgium

2 Research Foundation-Flanders (FWO), Brussels, Belgium

3 Department of Diagnostic Sciences, Ghent University, Ghent, Belgium a coagulopathy, consistent with a disseminated intravascular coagulopathy (DIC). In the extracellular compartment, monomeric globular actin (G-actin) polymerizes polymerized filamentous actin (F-actin), together with coagulation factor Va, which may cause vascular obstruction (DIC) and multiple organ dysfunction syndrome. To counteract the procoagulant effects of F-actin, the two members of the intravascular actin scavenging system, gelsolin, and DBP, cleave actin and inhibit repolymerization of G-actin [2]. However as demonstrated in a murine model, a lack of free DBP and increased concentrations and/or prolonged exposure to DBPactin complexes might induce endothelial cell injury and death, particularly in the lung microvasculature [4].

DBP binds to actin on the neutrophil plasma membrane, followed by elastase-mediated shedding of these complexes (perhaps as microvesicles) into the extracellular fluids. Until recently, DBP-actin complexes have been considered as benign by-products of cell injury. Recent evidence from in vitro and in vivo studies showed that DBP bound to G-actin, and not free DBP, functions as an indirect, but essential chemotactic cofactor for complement activation peptide $\mathrm{C} 5 \mathrm{a}$ and perhaps many leukocyte chemoattractants [5]. Using a mouse model, the complement system has been identified as an important host mediator of SARS-CoVinduced disease, which regulates the systemic proinflammatory response. Complement-deficient mice had a reduced neutrophilia in their lungs and reduced systemic inflammation [6]. As $25(\mathrm{OH})$ vitamin $\mathrm{D}_{3}$ and $1,25(\mathrm{OH})_{2}$ vitamin $\mathrm{D}_{3}$ inhibit the chemotaxis by competing for the same binding site on DBP [2], low vitamin D concentrations could be associated with a worse outcome of COVID-19.

Funding No source of funding was used. 


\section{Compliance with ethical standards}

Conflict of interest The authors declare that there is no conflict of interest regarding the publication of this letter.

Ethical approval Not applicable.

Informed consent For this article, a formal consent form is not required.

\section{References}

1. Ilie PC, Stefanescu S, Smith L (2020) The role of vitamin D in the prevention of coronavirus disease 2019 infection and mortality. Aging Clin Exp Res. https://doi.org/10.1007/s40520-020-01570 $-8$

2. Speeckaert MM, Speeckaert R, van Geel N et al (2014) Vitamin $\mathrm{D}$ binding protein: a multifunctional protein of clinical importance. Adv Clin Chem 63:1-57. https://doi.org/10.1016/b978-012-800094-6.00001-7
3. Du L, Zhou J, Zhang J et al (2012) Actin filament reorganization is a key step in lung inflammation induced by systemic inflammatory response syndrome. Am J Respir Cell Mol Biol 47:597-603. https ://doi.org/10.1165/rcmb.2012-0094OC

4. Ge L, Trujillo G, Miller EJ et al (2014) Circulating complexes of the vitamin $\mathrm{D}$ binding protein with G-actin induce lung inflammation by targeting endothelial cells. Immunobiology 219:198-207. https://doi.org/10.1016/j.imbio.2013.10.001

5. Kew RR (2019) The vitamin D binding protein and inflammatory injury: a mediator or sentinel of tissue damage? Front Endocrinol (Lausanne) 10:470. https://doi.org/10.3389/fendo.2019.00470

6. Gralinski LE, Sheahan TP, Morrison TE et al (2018) Complement activation contributes to severe acute respiratory syndrome coronavirus pathogenesis. mBio. https://doi.org/10.1128/mBio.01753 $-18$

Publisher's Note Springer Nature remains neutral with regard to jurisdictional claims in published maps and institutional affiliations. 